\title{
Costunolide induces G1/S phase arrest and activates mitochondrial-mediated apoptotic pathways in SK-MES 1 human lung squamous carcinoma cells
}

\author{
PEIYAN HUA ${ }^{1}$, GUANGXIN ZHANG ${ }^{1}$, YIFAN ZHANG $^{1}$, MEI SUN $^{2}$, \\ RANJI CUI ${ }^{3}, \mathrm{XIN} \mathrm{LI}^{3}$, BINGJIN LI $^{3}$ and XINGYI ZHANG ${ }^{1}$ \\ Departments of ${ }^{1}$ Thoracic Surgery and ${ }^{2}$ Pathology, and ${ }^{3}$ Jilin Provincial Key Laboratory of Molecular and Chemical Genetics, \\ The Second Hospital of Jilin University, Changchun, Jilin 130041, P.R. China
}

Received December 26, 2014; Accepted January 26, 2016

DOI: $10.3892 / \mathrm{ol} .2016 .4295$

\begin{abstract}
Despite the availability of several therapeutic options, a safer and more effective modality strategy is required for the treatment of lung cancer. Costunolide, a sesquiterpene lactone which isolated from the Saussurea lappa, has potent anticancer properties. In the present study, the effects of costunolide on cell viability, the cell cycle and apoptosis in SK-MES-1 human lung squamous carcinoma cells were investigated. Costunolide induced morphological changes and inhibited growth of SK-MES-1 cells growth. Flow cytometric analysis data demonstrated that costunolide significantly induced apoptosis of SK-MES-1 cells and induced cell cycle arrest at G1/S phase in a dose-dependent manner. Through upregulation in the expression of p53 and Bax, and downregulation in the expression of $\mathrm{Bcl}-2$ and activation of caspase-3, costunolide-induced apoptosis was confirmed by western blot analysis. In addition, the significant loss of mitochondrial membrane potential indicated that costunolide may induce apoptosis via the mitochondria-dependent pathway in SK-MES-1 cells. These results highlight the potential effects of costunolide as an anti-cancer agent in a human lung squamous carcinoma cell line.
\end{abstract}

\section{Introduction}

Lung cancer remains the leading cause of cancer-related mortality globally, with non-small-cell lung cancer (NSCLC)

Correspondence to: Professor Xingyi Zhang, Department of Thoracic Surgery, The Second Hospital of Jilin University, 218 Ziqiang Street, Changchun, Jilin 130041, P.R. China

E-mail: xingyizhang@live.com

Dr Bingjin Li, Jilin Provincial Key Laboratory of Molecular and Chemical Genetics, The Second Hospital of Jilin University, 218 Ziqiang Street, Changchun, Jilin 130041, P.R. China

E-mail: libingjin@jlu.edu.cn

Key words: costunolide, apoptosis, SK-MES-1 cells, G1/S phase accounting for $\sim 85 \%$ of total lung malignancies (1). At present, chemotherapy plays a critical role in the treatment of lung cancer (2). While the prevailing chemotherapy regimens can significantly suppress symptoms and improve quality of life for lung cancer patients, there is little effect on prolonging their overall survival $(3,4)$. Therefore, improved therapeutic options for lung cancer are urgently needed.

There is increasing evidence that phytochemicals from Chinese medicinal herbs may show promise as alternative therapeutic resources for treating malignancies (5-7). Sesquiterpene lactones are predominantly isolated from members of the Compositae and Magnoliaceae plant families, and have attracted widespread attention due to their anti-tumor and anti-inflammatory activity, $(8,9)$. Costunolide, a sesquiterpene lactone, which is isolated from Chinese herb Saussurea lappa, is a popular herbal remedy, with anti-ulcer (10), anti-inflammatory (11), anti-fungal (12) and anti-viral properties (13). Previous research has reported that costunolide can inhibit the expression of inducible nitric oxide synthase and the DNA-binding activity of NF- $\kappa \mathrm{B}(14,15)$. Furthermore, costunolide potentiates $1,25-(\mathrm{OH}) 2 \mathrm{D} 3$-induced differentiation in HL-60 promyelocytic leukemia cells by modulating NF- $\kappa \mathrm{B}$ activation $(16,17)$. Previous studies have demonstrated that costunolide has anti-tumor potential by inhibiting proliferation, inducing apoptosis and reducing invasion and metastasis in a number of tumor cells including intestinal neoplasia and melanoma, leukemia and hepatocellular carcinoma cells, and cervical, prostate, bladder, colon and breast cancer cells $(6,18-25)$. The effects of costunolide on human lung squamous carcinoma cells are still unknown. The present study aimed to elucidate the effects of costunolide on the proliferation of SK-MES-1 cells, and to explore the possible mechanism of costunolide-induced apoptosis in this lung cancer cell line, in order to assess its potential as a future therapeutic option for lung cancer patients.

\section{Materials and methods}

Materials. The SK-MES-1 human lung squamous carcinoma cell line was a gift from Dr. T.H. Ma (Jilin University Bethune Second Hospital, Changchun, China). Costunolide 
was purchased from the Tongtian (Shanghai, China), and was dissolved in dimethyl sulfoxide (DMSO), purchased from Shenggong (Shanghai, China) to make a stock solution. Fetal bovine serum (FBS) was purchased (Gibco; Thermo Fisher Scientific, Inc., Waltham, MA, USA). 3-(4,5-dimethylthiazol-2-yl)-2,5-diphenyltetrazolium bromide (MTT), Hoechst 33342, Dulbecco's Modified Eagle's Medium (DMEM) and rhodamine-123 mitochondrial specific fluorescent dye were purchased from Sigma-Aldrich, St. Louis, MO, USA. The cell cycle analysis and reactive oxygen species assay kits were purchased from Beyotime Institute of Biotechnology (Shanghai, China). BCA protein assay kit and Annexin V-FITC apoptosis detection kit were purchased from Nanjing KeyGen Biotech Co., Ltd., (Nanjing, China). Polyclonal anti-mouse antibodies raised against $\beta$-actin (catalog no., AA128; dilution, 1:2,000), p21 (catalog no., AP021; dilution, 1:500), p27 (catalog no., AP027; dilution, 1:500) and phospho-Rb (catalog no., AR092); dilution, 1:1,000), as well as horseradish peroxidase (HRP)-conjugated secondary antibodies (goat anti-mouse IgG; catalog no., A0216; dilution, 1:1,000) were purchased from Beyotime Institute of Biotechnology. Polyclonal antibodies raised against Bax (catalog no., 2772S; dilution, 1:1,000), Bcl-2 (catalog no., 2876S; dilution, 1:1,000), pro-caspase-3 (catalog no., 9662P; dilution, 1:1,000), p53 (catalog no., 9282S; dilution, 1:1,000), poly-ADP-ribose polymerase (PARP; catalog no., 9542S; dilution, 1:1,000) and horseradish peroxidase-conjugated secondary antibodies (goat anti-rabbit IgG; catalog no., 7074P2; dilution, 1:2,000) were purchased from Cell Signaling Technology, Inc., Shanghai, China. Western Blotting detection kit was purchased from EMD Millipore (Billerica, MA, USA).

Cell culture. Human lung squamous carcinoma SK-MES-1 cells were cultured in DMEM nutrients mixture supplemented with $10 \% \mathrm{FBS}$ at $37^{\circ} \mathrm{C}$ in a humidified atmosphere with $5 \% \mathrm{CO}_{2}$ Cells were cultured in $10 \mathrm{~cm}$ culture dishes and allowed to reach $\sim 70 \%$ confluence before being used in experiments.

Cell growth inhibition assay. The cytotoxic effects of the costunolide on the cell were determined using the MTT assay. Briefly, SK-MES-1 cells were seeded at a density of $1 \times 10^{4}$ cells/well in 96-well plates and incubated overnight. Cells were treated with drug monomer with different concentrations. Each of the total incubation volume well was $100 \mu \mathrm{l}$. Following $24 \mathrm{~h}$ of incubation, $10 \mu \mathrm{l}$ MTT was added to each well and incubated for an additional $4 \mathrm{~h}$. The supernatants were then removed and $150 \mu \mathrm{l}$ DMSO was added to dissolve the formazan crystals. In this assay, viable cell number is directly proportional to the production of formazan. Absorbance was then read in a Varioskan ${ }^{\mathrm{TM}}$ Flash Multimode Reader (Thermo Scientific) at a wavelength of $570 \mathrm{~nm}$. The assay was repeated three times. The half maximal inhibitory concentration $\left(\mathrm{IC}_{50}\right)$ values were calculated using GraphPad Prism 5 (GraphPad Software, Inc., La Jolla, CA, USA)

The percentage of inhibition, the inhibitory ratio (IR), was calculated in the present study using the following formula: $\operatorname{IR}(\%)=\left(\mathrm{A}_{570}\right.$ [control] $-\mathrm{A}_{570}$ [sample] $) / \mathrm{A}_{570}$ [control] $\mathrm{x} 100 \%$.

Flow cytometric cell cycle analysis. Cell cycle analysis was detected by flow cytometry using a propidium iodide (PI) cell cycle detection kit (catalog no., C1052; Beyotime Institute of Biotechnology). Briefly, SK-MES-1 cells were seeded into 6-well plates and incubated overnight. Costunolide at concentrations of 0,40 and $80 \mu \mathrm{M}$ was added to the wells and incubated for a further $24 \mathrm{~h}$. Cells were then harvested and fixed in $500 \mu 170 \%$ ice-cold ethanol at $4^{\circ} \mathrm{C}$ for $2 \mathrm{~h}$. Then, samples were washed with phosphate-buffered saline (PBS) and incubated with RNase A and PI staining solution, according to the manufacturer's instructions.

Nuclei fragmentation analysis by Hoechst 33342 staining. SK-MES-1 cells were treated with 0,40 and $80 \mu \mathrm{M}$ costunolide for $24 \mathrm{~h}$. The cells were fixed with $4 \%$ paraformaldehyde for $30 \mathrm{~min}$ at room temperature. After washing with PBS, the cells were stained with Hoechst $33342(10 \mu \mathrm{g} / \mathrm{ml})$ at $37^{\circ} \mathrm{C}$ for $20 \mathrm{~min}$ in the dark. Finally, the cells were washed and resuspended in PBS for the observation of nuclear morphology using an Olympus 1X71 fluorescence microscope (Olympus Corp., Tokyo, Japan).

Apoptosis analysis by flow cytometry using annexin-PI staining. The apoptosis of SK-MES-1 cells was assessed by flow cytometry using annexin V-FITC/PI staining. Briefly, the SK-MES-1 cells were seeded into 6-well plates and incubated overnight. Costunolide at concentrations of 0,40 and $80 \mu \mathrm{M}$ was added to the cells and incubated for $24 \mathrm{~h}$. The cells were then collected, washed, and resuspended in PBS. The apoptotic cell death rate was examined by Annexin V-FITC and PI double staining using the Annexin V-FITC apoptosis detection kit (catalog no., KGA105; Nanjing KeyGen Biotech Co., Ltd.), according to the manufacturer's instructions. Following the Annexin V and PI staining, the cells were subjected to flow cytometric analysis and data were analyzed using Cell Quest software.

Flow cytometric determination of mitochondrial membrane potential $(\Delta \Psi m)$. Rhodamine 123 was used to evaluate perturbations in mitochondrial transmembrane potential in SK-MES-1 cells by flow cytometry. Briefly, SK-MES-1 cells were plated in 6-well dishes and cells were treated with 0 , 40 and $80 \mu \mathrm{M}$ of costunolide for $24 \mathrm{~h}$. Cells were collected in centrifuge tube and resuspended in $500 \mu \mathrm{l} \mathrm{PBS}$, and then incubated with the rhodamine $123\left(10 \mu \mathrm{M}\right.$; Sigma-Aldrich) at $37^{\circ} \mathrm{C}$ for $20 \mathrm{~min}$. Cells were centrifuged at $1500 \mathrm{rpm}$ for $5 \mathrm{~min}$, the supernatant was removed, and cell pellets were gently rinsed once with PBS, then resuspended in $200 \mu 1$ PBS. Following filtration, the suspension was analyzed by flow cytometry.

Western blot analysis of protein expression. To evaluate the effect of costunolide and reveal the mechanism of apoptosis induction, western blotting was used to measure the expression levels of apoptosis-related proteins. Firstly, SK-MES-1 cells were treated with 0,40 and $80 \mu \mathrm{M}$ of costunolide for $24 \mathrm{~h}$. Both adherent and floating cells were collected in $15 \mathrm{ml}$ centrifuge tubes and washed with PBS. The cell pellets were resuspended in RIPA lysis buffer and were then ultrasound lysed on ice. After centrifugation for $5 \mathrm{~min}$, the supernatant was collected and the protein content of the supernatant was determined using a bicinchoninic acid assay (BCA) protein assay kit, and the protein samples were stored at $-80^{\circ} \mathrm{C}$. Protein lysates were separated by electrophoresis on a $10 \%$ sodium dodecyl sulfate (SDS)-polyacrylamide gel and transferred to a polyvinylidene 

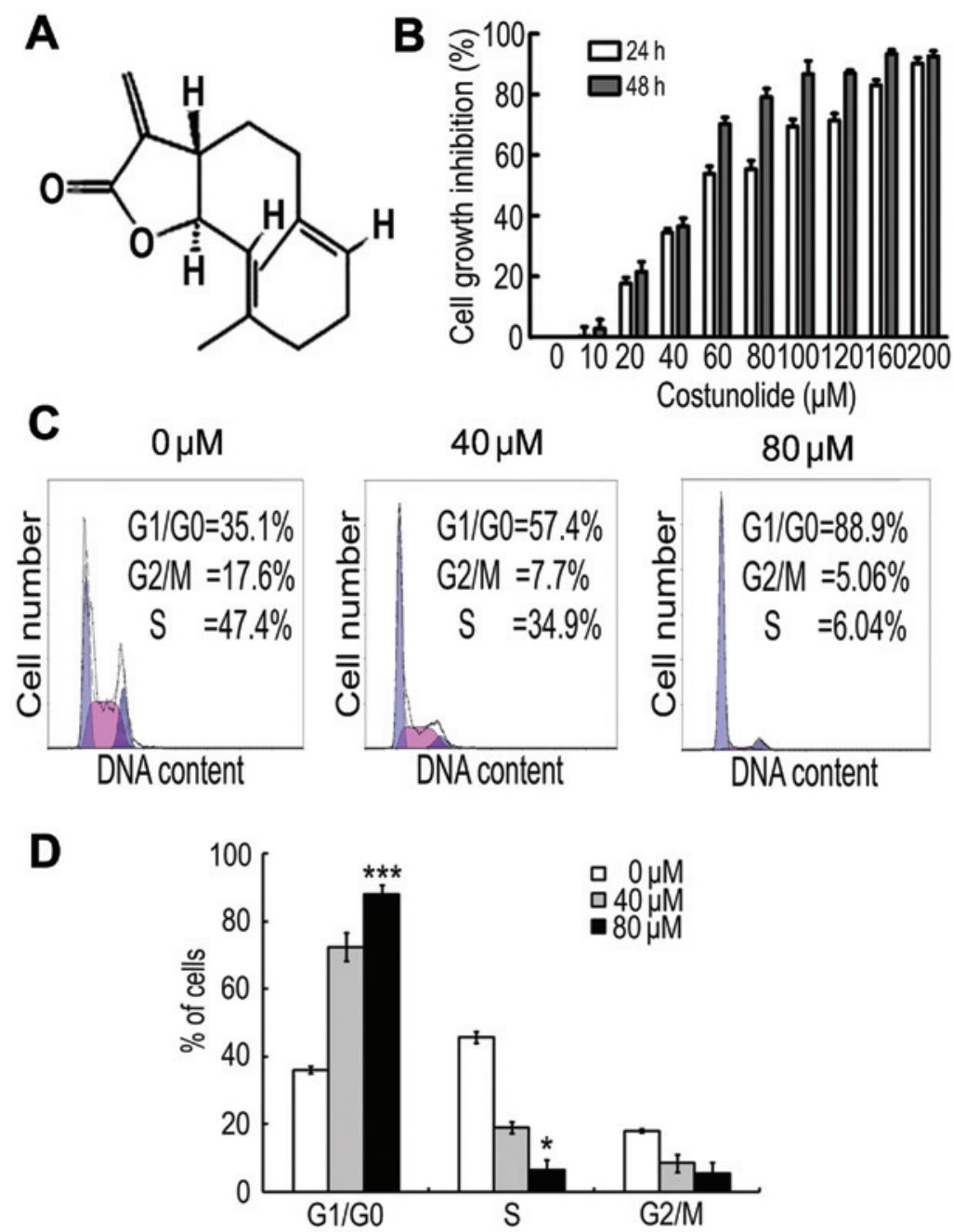

Figure 1. (A) Chemical structures of costunolide. (B) Costunolide-induced growth inhibition of SK-MES-1 cells. The cells were exposed to the indicated concentrations of costunolide for 24 and $48 \mathrm{~h}$, and the percentages of growth inhibition and viability were measured. Data are expressed as mean \pm SD of three independent experiments. (C) Effect of costunolide on cell cycle distribution. SK-MES- 1 cells were treated with 0,40 and $80 \mu \mathrm{M}$ of costunolide for $24 \mathrm{~h}$ and then stained with propidium iodide for flow cytometric analysis. Histograms show the numbers of cells/channel (y-axis) vs. DNA content (x-axis). Values indicate the percentages of cells in the corresponding phases of cell cycle. (D) Data are expressed as mean \pm SD of three independent experiments. Columns not sharing the same superscript letter differ significantly, ${ }^{* * *} \mathrm{P}<0.01$ and ${ }^{*} \mathrm{P}<0.05$ compared to the control.

fluoride membrane. The membranes were then soaked in blocking buffer (5\% skimmed milk) for $1 \mathrm{~h}$. To probe for the proteins of interest, membranes were incubated overnight at $4^{\circ} \mathrm{C}$ with the relevant aforementioned antibodies, followed by appropriate HRP-conjugated secondary antibodies and enhanced chemiluminescence reagents (catalog no., WBKLS0100; EMD Millipore). A Gel-Pro Analyzer (Gel-Pro 32, version 4.0; Media Cybernetics, Inc., Rockville, MD, USA) was used to extract qualitative and quantitative information from the electrophoretic gels to document and store the western blot data.

Statistical analysis. Data were expressed as the mean \pm standard deviation. Comparisons were made using a one-way ANOVA, followed by Dunnett's test. $\mathrm{P}<0.05$ was considered to indicate a statistically significant difference.

\section{Results}

Effect of costunolide on SK-MES-1 cell proliferation. To detect the effect of costunolide (structure shown in Fig. 1A) on the proliferation of SK-MES-1 cells, an MTT assay was performed. The results demonstrate that costunolide reduced cell viability in a time- and dose-dependent manner (Fig. 1B). The $\mathrm{IC}_{50}$ values were $\sim 60 \mu \mathrm{M}$ after $24 \mathrm{~h}$ of treatment, and $\sim 50 \mu \mathrm{M}$ following $48 \mathrm{~h}$ of costunolide treatment.

Costunolide induces cell cycle arrest in SK-MES-1 cells. Cell cycle arrest is one of the major causes of cell growth inhibition. In order to find out whether cell growth inhibition was due to cell cycle arrest at a specific phase of cell cycle, the cell cycle profile was determined by PI staining and flow cytometry analysis. The results demonstrate that costunolide arrested the cell cycle at G1/S phase in a dose-dependent manner (Fig. 1C).

Costunolide induces apoptotic cell death in SK-MES-1 cells. DNA fragmentation and loss of plasma membrane asymmetry are the major features of apoptotic cell death. The effect of costunolide on cell death was analyzed by observing the nuclear morphological changes using Hoechst 33342 staining 

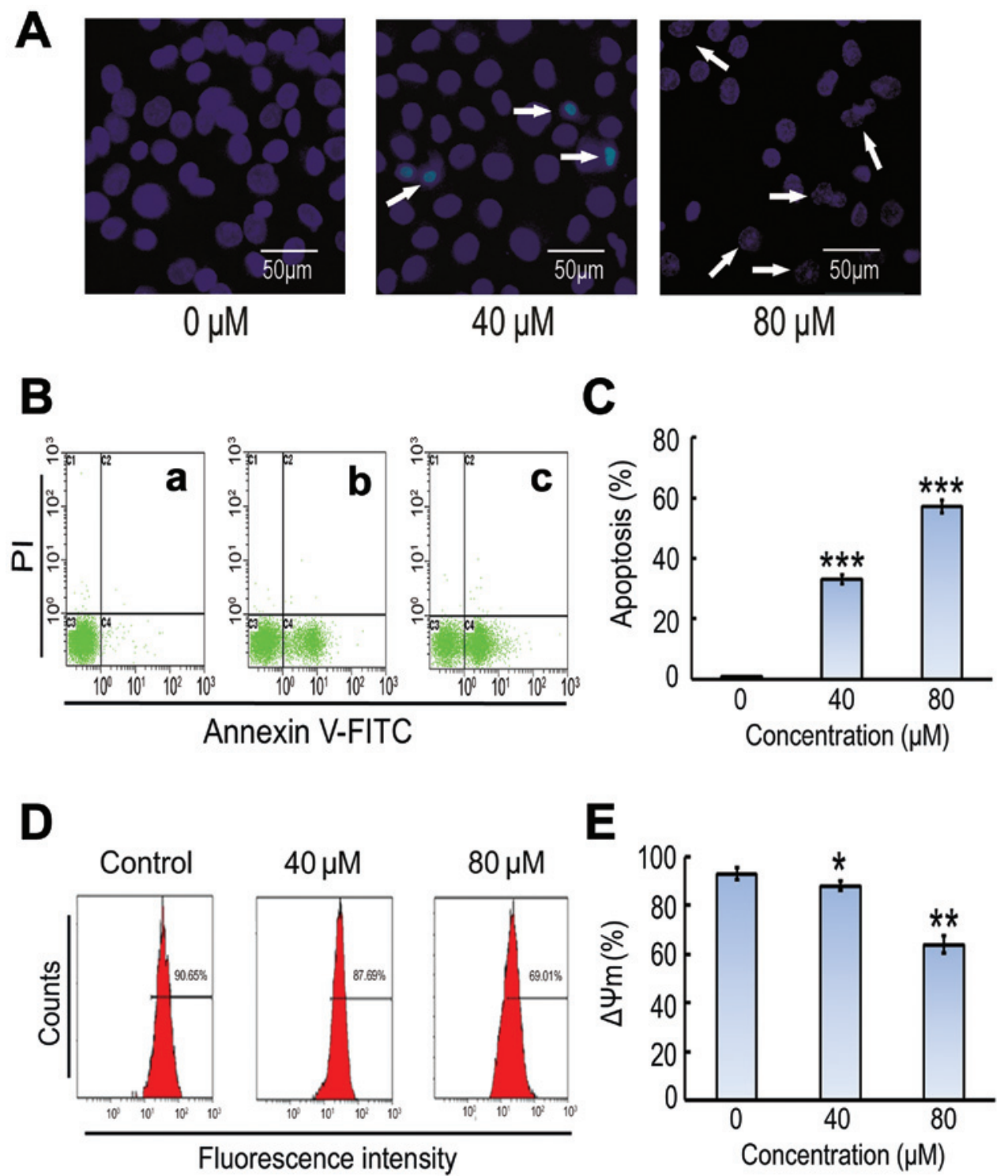

Figure 2. (A) Induction of apoptosis by costunolide in SK-MES-1 cells, detected using Hoechst 33342 staining. SK-MES-1 cells were treated with 40 and $80 \mu$ M costunolide for $24 \mathrm{~h}$ and nuclear morphological changes were imaged using fluorescent microscopy. Apoptotic changes, such as condensed chromatin and fragmented nuclei are indicated by arrows. (B) Apoptosis induced by costunolide in SK-MES- 1 cells. SK-MES- 1 cells were treated with (a) $0 \mu \mathrm{M}$, (b) $40 \mu \mathrm{M}$ and (c) $80 \mu \mathrm{M}$ costunolide for $24 \mathrm{~h}$, then stained with FITC-conjugated Annexin V and PI using flow cytometric analysis. (C) Quantification of cell apoptosis. Data are expressed as mean \pm SD of three independent experiments. ${ }^{* * *} \mathrm{P}<0.001$ compared to the control. (D) The effects of costunolide on mitochondrial transmembrane potential of SK-MES-1 cells were determined by flow cytometry. The values indicate the percentages of rhodamine 123 fluorescence in the SK-MES-1 cells treated without (control) and with $40 \mu \mathrm{M}$ and $80 \mu \mathrm{M}$ of costunolide for $24 \mathrm{~h}$. The data shown are representative of three independent experiments with the similar results. (E) Data are expressed as mean \pm SD of three independent experiments. Columns not sharing the same superscript letter differ significantly, ${ }^{*} \mathrm{P}<0.05$ and ${ }^{* *} \mathrm{P}<0.01$ compared with the control.

and fluorescent microscopy. As shown in Fig. 2A, costunolide induced obvious nuclear morphological changes, including nuclear shrinkage and DNA fragmentation in SK-MES-1 cells in a dose-dependent manner. Induction of apoptosis was further confirmed by Annexin V-FITC and PI staining (Fig. 2B).

Costunolide induces apoptosis in SK-MES-1 cells and mitochondrial membrane potential. To further investigate costunolide-induced inhibitory effect, SK-MES-1 cells were treated with costunolide as described in the methods section, and the percentage of cells undergoing apoptosis or necrosis was determined using flow cytometric analysis with annexin V-FITC and PI staining. The results demonstrate that costunolide treatment induced apoptosis in a dose-dependent manner (Fig. 2B). A significant increase was observed in early apoptosis in experimental group compared to control group (Fig. 2B and C). The effects of costunolide on the mitochondrial membrane potential of SK-MES-1 cells were determined by flow cytometry using rhodamine 123 staining. The results demonstrated that rates of depletion of mitochondrial membrane potential were reduced following costunolide treatment (Fig. 2D and E).

Effect of costunolide on the expression of cell cycle regulators. To elucidate the molecular mechanism underlying G1/S phase arrest induced by costunolide, the key proteins involved in the regulation of $\mathrm{G} 1 / \mathrm{S}$ transition in SK-MES-1 cells 

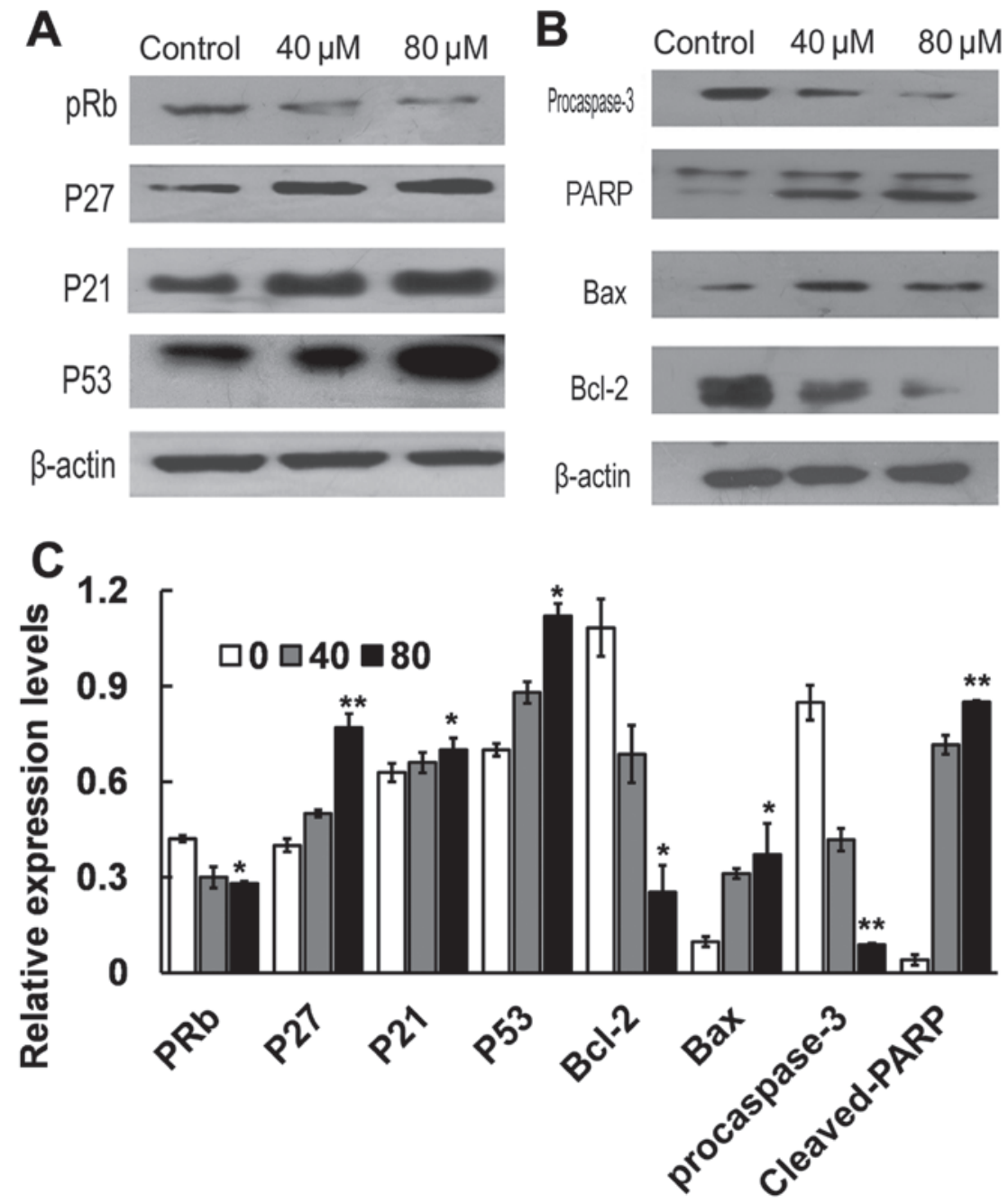

Figure 3. (A) Effect of costunolide on the expression of cell cycle regulators. SK-MES-1 cells were treated with 0,40 and $80 \mu \mathrm{M}$ costunolide for $24 \mathrm{~h}$. The expression of p53, p21, p27 and phospho-retinoblastoma (pRb) were determined by western blot analysis. (B) Effect of costunolide on the expression of apotosis regulators. SK-MES-1 cells were treated with 0,40 and $80 \mu \mathrm{M}$ costunolide for $24 \mathrm{~h}$, respectively. The expression of Bcl-2, Bax, Caspase 3 and PARP were determined by western blot analysis. (C) Data are expressed as mean \pm SD of three independent experiments. Columns not sharing the same superscript letter differ significantly, ${ }^{*} \mathrm{P}<0.05$ and ${ }^{* *} \mathrm{P}<0.01$ compared with the control.

were investigated. Cells were treated with different doses of costunolide $(40,80 \mu \mathrm{M})$ for $24 \mathrm{~h}$.The expression of $\mathrm{p} 53$, p21, p27 and pRb proteins were analyzed by western blotting. Treatment with costunolide led to upregulation the expression of p53, p27 and p21, decreased the expression of pRb in a dose-dependent manner in SK-MES-1 cells (Fig. 3A, C).

Effect of costunolide on the expression of apoptosis regulators. The results of the present study demonstrated that costunolide increases the protein expression levels of $\mathrm{p} 53$, which is related to $\mathrm{Bcl}-2$ protein family. The expression of Bax and $\mathrm{Bcl}-2$ was examined by western blot in SK-MES- 1 cells treated with 0,40 and $80 \mu \mathrm{M}$ of costunolide for $24 \mathrm{~h}$. The results demonstrated that the expression of Bax was markedly increased following treatment with costunolide, accompanied with decreased expression of Bcl-2 in a dose-dependent manner. Next, we examined the effect of costunolide on caspase-3 activation by western blot analysis. The results demonstrated that expression of cleaved PARP ( $85 \mathrm{kDa}$ fragment) and procaspase 3 were decreased following costunolide treatment (Figs. 3B and C).

\section{Discussion}

The aim of the present study was to identify a novel therapeutic agent to target lung cancer. Sesquiterpene lactones are phytochemicals derived from Chinese medicinal herbs, and have been demonstrated previously to have anti-neoplastic and anti-inflammatory activities (8). Costunolide is a well-known sesquiterpene lactone and a number of previous studies have shown that it has a broad spectrum of cytotoxicity against human cancer cell lines of different origins $(6,18,20-22,26,27)$.

In the present study, the inhibitory effect of costunolide on the proliferation of lung cancer cells SK-MES-1 was investigated in vitro. The results of the MTT assay demonstrated that costunolide reduced cell viability in a time- and dose-dependent manner (Fig. 1B). Previous studies have revealed that cell cycle arrest and apoptosis are two mechanisms involved in the induction of cell death (28). Studies on cell cycle regulation have shown that cell cycle progression is tightly controlled by various checkpoints in normal cells while alterations in the checkpoints of cell cycle progression lead to aberrant cell proliferation and development of cancer (29). Tumor cells frequently acquiring defects in the 
checkpoints leads to unrestrained proliferation (30). Many anti-tumor drugs induce cell cycle arrest at a specific checkpoint and thereby induce apoptosis $(31,32)$. The present study identified that costunolide-induced cell cycle arrest at G1/S phase is accompanied by a reduction in $\mathrm{G} 2 / \mathrm{M}$ and $\mathrm{S}$ phase in a dose-dependent manner by using flow cytometric analysis. These findings are in line with other reports (21). Furthermore, the current study provided evidence that G1/S phase cell cycle arrest is one of the mechanisms in the growth inhibitory effect of costunolide in SK-MES-1 cells. In addition to cell cycle arrest, costunolide exerts its cytotoxic effects via the induction of apoptosis in SK-MES-1 cells. These data strongly suggested that the cytotoxic effect of costunolide in SK-MES-1 cells via induction of apoptosis, and in agreement with previous studies, induction of other cancer cells including leukemia (16), prostate cancer cells (21), ovarian cancer cells (27) and bladder cancer cells (6).

P53, a tumor suppressor protein, plays a key role in the regulation of cell cycle progression, checkpoint activation and apoptosis $(33,34)$. The data presented herein suggest that costunolide treatment upregulates the expression of $\mathrm{p} 53$ protein and increases the expression of $\mathrm{p} 21$, a downstream target of p53. The cell cycle dependent kinase inhibitor p21, one of the Clp family members, is located downstream of the p53 gene. Cell cycle proteins p21 and protein kinase $2 / \mathrm{E}$, lead to inhibiting the activity of the complexes and retinoblastoma protein ( $\mathrm{Rb}$ ) phosphorylation. Rb cannot release the E2F subunit, which participate in DNA synthesis (35). As a result, cell cycle arrested in G1 phase. In addition, p27 is a cyclin-dependent kinase inhibitor which controls G1/S transition by inhibiting the activity of a wide variety of cyclin/cyclin dependent kinase (CDK) complex (36). In the present study, costunolide-treated SK-MES-1 cells decreased in the expression of p27. Our data showed that the costunolide-mediated G1/S phase cell cycle arrest in SK-MES-1 cells was associated with the increase expression of $\mathrm{p} 27$. These findings may explain in part the mechanisms underlying G1/S phase arrest, and further studies are required to fully elucidate these molecular mechanisms.

Many reports have shown that p53 is a tumor suppressor protein which triggers apoptosis by inducing mitochondrial membrane permeabilization through regulating the expression of apoptosis mediated proteins $(37,38)$. The $\mathrm{Bcl}-2$ protein family is a large family of apoptosis regulating proteins that modulate the mitochondrial pathway and includes anti-apoptotic proteins and pro-apoptotic proteins such as Bcl-2 and Bax (39). To explore the further molecular mechanisms underpinning costunolide-induced apoptosis in SK-MES-1 cells, the expression of Bax and Bcl-2 protein in SK-MES-1 cells of each group was examined. The results demonstrated that the expression of Bax gradually increased and Bcl-2 decreased in treatment groups in a dose-dependent manner. Taken together, these data demonstrate that p53 plays a critical role in costunolide-mediated apoptosis in SK-MES-1 cells. $\geq$

As the expression of Bax increases, a significant reduction in mitochondrial transmembrane potential is observed in the cells of treatment groups. Mitochondrial permeability transition pores are opened, which lead to the release of cytochrome $\mathrm{C}$ and other pro-apoptotic molecules from intermembranous space to cytosol, activating downstream caspases and ultimately caspase 3 (40). Caspase 3 is a frequently activated death protease which cleave PARP, a DNA repair enzyme (41). The present study demonstrated the cleavage of PARP into its $85 \mathrm{kDa}$ fragment and the decreased expression of procaspase 3 . Our results clearly demonstrate that the mitochondrial-mediated caspase activation pathway is involved in costunolide-mediated apoptosis in SK-MES-1cells.

While the mechanisms of the growth inhibitory effect of costunolide in some cancer cells have previously been demonstrated $(6,19,25,27)$, our study is the first time to describe this in human lung squamous carcinoma cells. In conclusion, costunolide induced apoptosis in SK-MES-1 cells accompany with a marked loss of G1/S phase cells. Costunolide-induced apoptosis marked with upregulation of Bax and p53, downregulation of $\mathrm{Bcl}-2$ and caspase- 3 with cleaved PARP in a dose-dependent manner. These findings identify that costunolide may become a potential therapeutic target for the future development of anti-lung cancer agents.

\section{Acknowledgements}

The present study was supported by grants from the Science and Technology Services of Jilin Province Scientific and Technological Project (grant no. 20140521) and the Natural Science Foundation of China (grant no. 81272472).

\section{References}

1. Jemal A, Siegel R, Ward E, Murray T, Xu J, Smigal C and Thun MJ: Cancer statistics, 2006. CA Cancer J Clin 56: 106-130, 2006.

2. Saba NF and Khuri FR: Chemoprevention strategies for patients with lung cancer in the context of screening. Clin Lung Cancer 7: 92-99, 2005.

3. Chou JY, Lai SY, Pan SL, Jow GM, Chern JW and Guh JH: Investigation of anticancer mechanism of thiadiazole-based compound in human non-small cell lung cancer A549 cells. Biochem Pharmacol 66: 115-124, 2003.

4. Bonomi P, Kim K, Fairclough D, Cella D, Kugler J, Rowinsky E, Jiroutek M and Johnson D: Comparison of survival and quality of life in advanced non-small-cell lung cancer patients treated with two dose levels of paclitaxel combined with cisplatin versus etoposide with cisplatin: Results of an Eastern Cooperative Oncology Group trial. J Clin Oncol 18: 623-631, 2000.

5. Lin Y, Xu J, Liao H, Li L and Pan L: Piperine induces apoptosis of lung cancer A549 cells via p53-dependent mitochondrial signaling pathway. Tumour Biol 35: 3305-3310, 2014.

6. Rasul A, Bao R, Malhi M,Zhao B, Tsuji I, Li J and Li X: Induction of apoptosis by costunolide in bladder cancer cells is mediated through ROS generation and mitochondrial dysfunction. Molecules 18: 1418-1433, 2013.

7. Bonomi P: Review of paclitaxel/carboplatin in advanced non-small cell lung cancer. Semin Oncol 26 (Suppl 2): S55-S59, 1999.

8. Gu JQ, Gills JJ, Park EJ, Mata-Greenwood E, Hawthorne ME, Axelrod F, Chavez PI, Fong HH, Mehta RG, Pezzuto JM and Kinghorn AD: Sesquiterpenoids from Tithonia diversifolia with potential cancer chemopreventive activity. J Natl Prod 65: 532-536, 2002.

9. Koch E, Klaas CA, Rüngeler P, Castro V, Mora G, Vichnewski W and Merfort I: Inhibition of inflammatory cytokine production and lymphocyte proliferation by structurally different sesquiterpene lactones correlates with their effect on activation of NF-kappaB. Biochem Pharmacol 62: 795-801, 2001.

10. Robles M, Aregullin M, West J and Rodriguez E: Recent studies on the zoopharmacognosy, pharmacology and neurotoxicology of sesquiterpene lactones. Planta Med 61: 199-203, 1995.

11. Park HJ, Jung WT, Basnet P, Kadota $S$ and Namba T: Syringin 4-O-beta-glucoside, a new phenylpropanoid glycoside and costunolide, a nitric oxide synthase inhibitor, from the stem bark of Magnolia sieboldii. J Nat Prod 59: 1128-1130, 1996.

12. Wedge DE, Galindo JC and Macías FA: Fungicidal activity of natural and synthetic sesquiterpene lactone analogs. Phytochemistry 53: 747-757, 2000 . 
13. Chen HC, Chou CK, Lee SD, Wang JC and Yeh SF: Active compounds from Saussurea lappa Clarks that suppress hepatitis $\mathrm{B}$ virus surface antigen gene expression in human hepatoma cells. Antiviral Res 27: 99-109, 1995.

14. Koo TH, Lee JH, Park YJ, Hong YS, Kim HS, Kim KW and Lee JJ: A sesquiterpene lactone, costunolide, from Magnolia grandiflora inhibits NF-kappa B by targeting I kappa B phosphorylation. Planta Med 67: 103-107, 2001.

15. Fukuda K, Akao S, Ohno Y, Yamashita K and Fujiwara H: Inhibition by costunolide of phorbol ester-induced transcriptional activation of inducible nitric oxide synthase gene in a human monocyte cell line THP-1. Cancer Lett 164: 7-13, 2001.

16. Choi JH, Ha J, Park JH, Lee JY, Lee YS, Park HJ, Choi JW, Masuda Y, Nakaya K and Lee KT: Costunolide triggers apoptosis in human leukemia U937 cells by depleting intracellular thiols. Jpn J Cancer Res 93: 1327-1333, 2002.

17. Kim SH, Kang SN, Kim HJ and Kim TS: Potentiation of 1,25-dihydroxyvitamin $\mathrm{D}(3)$-induced differentiation of human promyelocytic leukemia cells into monocytes by costunolide, a germacranolide sesquiterpene lactone. Biochem Pharmacol 64: 1233-1242, 2002.

18. Choi YK, Cho SG, Woo SM, Yun YJ, et al: Saussurea lappa clarke-derived costunolide prevents TNF $\alpha$-induced breast cancer cell migration and invasion by inhibiting NF- $\kappa \mathrm{B}$ activity. Evid Based Complement Alternat Med 2013: 936257, 2013.

19. Choi YK, Seo HS, Choi HS, Choi HS, Kim SR, Shin YC and Ko SG: Induction of Fas-mediated extrinsic apoptosis, p21WAF1-related G2/M cell cycle arrest and ROS generation by costunolide in estrogen receptor-negative breast cancer cells, MDA-MB-231. Mol Cell Biochem 363: 119-128, 2012.

20. Liu CY, Chang HS, Chen IS, Chen CJ,Hsu ML, Fu SL and Chen YJ: Costunolide causes mitotic arrest and enhances radiosensitivity in human hepatocellular carcinoma cells. Radiat Oncol 6: 56, 2011.

21. Hsu JL, Pan SL, Ho YF, Hwang TL, Kung FL and Guh JH: Costunolide induces apoptosis through nuclear calcium2+ overload and DNA damage response in human prostate cancer. $\mathrm{J}$ Urol 185: 1967-1974, 2011.

22. Choi JH and Lee KT: Costunolide-induced apoptosis in human leukemia cells: Involvement of c-jun N-terminal kinase activation. Biol Pharm Bull 32: 1803-1808, 2009.

23. Choi SH, Im E, Kang HK, Lee JH, Kwak HS, Bae YT, Park HJ and Kim ND: Inhibitory effects of costunolide on the telomerase activity in human breast carcinoma cells. Cancer Lett 227: 153-162, 2005.

24. KomiyaT, Yamada Y,MotekiH,KatsuzakiH,ImaiKandHibasamiH: Hot water soluble sesquiterpenes [anhydroperoxy-costunolide and 3-oxoeudesma-1,4 (15),11 (13)triene-12,6alpha-olide] isolated from laurel (Laurus nobilis L.) induce cell death and morphological change indicative of apoptotic chromatin condensation in leukemia cells. Oncol Rep 11: 85-88, 2004.

25. Hibasami H, Yamada Y, Moteki H, Katsuzaki H,Imai K, Yoshioka K and Komiya T: Sesquiterpenes (costunolide and zaluzanin D) isolated from laurel (Laurus nobilis L.) induce cell death and morphological change indicative of apoptotic chromatin condensation in leukemia HL-60 cells. Int J Mol Med 12: 147-151, 2003.
26. Kretschmer N, Rinner B, Stuendl N, Kaltenegger H, Wolf E, Kunert O, Boechzelt H, Leithner A, Bauer R and Lohberger B: Effect of costunolide and dehydrocostus lactone on cell cycle, apoptosis and $\mathrm{ABC}$ transporter expression in human soft tissue sarcoma cells. Planta Med 78: 1749-1756, 2012.

27. Yang YI, Kim JH and Choi JH: Costunolide induces apoptosis in platinum-resistant human ovarian cancer cells by generating reactive oxygen species. Gynecol Oncol 123: 588-596, 2011.

28. King KL and Cidlowski JA: Cell cycle regulation and apoptosis. Ann Rev Physiol 60: 601-617, 1998.

29. Collins K, Jacks T and Pavletich NP: The cell cycle and cancer. Proc Natl Acad Sci USA 94: 2776-2778, 1997.

30. Pan J, She M, Xu ZX, Sun L and Yeung SC: Farnesyltransferase inhibitors induce DNA damage via reactive oxygen species in human cancer cells. Cancer Res 65: 3671-3681, 2005.

31. Bergamo A, Gagliardi R, Scarcia V, Furlani A, Alessio E, Mestroni G and Sava G: In vitro cell cycle arrest, in vivo action on solid metastasizing tumors and host toxicity of the antimetastatic drug NAMI-A and cisplatin. J Pharmacol Exp Ther 289: 559-564, 1999 .

32. Horwitz SB: Taxol (paclitaxel): Mechanisms of action. Ann Oncol 5 (Suppl 6): S3S6, 1994.

33. Fridman JS and Lowe SW: Control of apoptosis by p53. Oncogene 22: 9030-9040, 2003.

34. Budram-Mahadeo V, Morris PJ and Latchman DS: The Brn-3a transcription factor inhibits the pro-apoptotic effect of p53 and enhances cell cycle arrest by differentially regulating the activity of the p53 target genes encoding Bax and p21(CIP1/Waf1). Oncogene 21: 6123-6131, 2002.

35. Tchernev G and Orfanos CE: Downregulation of cell cycle modulators p21, p27, p53, Rb and proapoptotic Bcl-2-related proteins Bax and Bak in cutaneous melanoma is associated with worse patient prognosis: Preliminary findings. J Cutan Pathol 34: 247-256, 2007

36. Polyak K, Kato JY, Solomon MJ, Sherr CJ, Massague J, Roberts JM and Koff A: p27Kip1, a cyclin-Cdk inhibitor, links transforming growth factor-beta and contact inhibition to cell cycle arrest. Genes Dev 8: 9-22, 1994

37. Rasul A, Ding C, Li X, Khan M, Yi F, Ali M and Ma T: Dracorhodin perchlorate inhibits PI3K/Akt and NF- $\kappa B$ activation, up-regulates the expression of p53 and enhances apoptosis. Apoptosis 17: 1104-1119, 2012.

38. Sakuragi N, Salah-eldin AE, Watari H, Itoh T, Inoue $\mathrm{S}$, Moriuchi $\mathrm{T}$ and Fujimoto S: Bax, Bcl-2 and p53 expression in endometrial cancer. Gynecol Oncol 86: 288-296, 2002.

39. Frenzel A, Grespi F, Chmelewskij W and Villunger A: $\mathrm{Bcl} 2$ family proteins in carcinogenesis and the treatment of cancer. Apoptosis 14: 584-596, 2009.

40. Ly JD, Grubb DR and Lawen A: The mitochondrial membrane potential (deltapsi(m)) in apoptosis; an update. Apoptosis 8: $115-128,2003$.

41. Porter AG and Jünicke RU: Emerging roles of caspase-3 in apoptosis. Cell Death Differ 6: 99-104, 1999. 